\title{
ОЦЕНКА ЭФФЕКТИВНОСТИ ПУТЕЙ ВЫЯВЛЕНИЯ ОПУХОЛЕЙ ЖЕНСКИХ ПОЛОВЫХ ОРГАНОВ НА УРОВНЕ ПЕРВИЧНОГО ЗВЕНА ЗДРАВООХРАНЕНИЯ
}

\author{
А. Г. Бочкова ${ }^{1 凶}$, А. С. Доможирова², И. А. Аксенова ${ }^{1,3}$
}

1 Южно-Уральский государственный медицинский университет, Челябинск, Россия

2 Российский научный центр рентгенорадиологии, Москва, Россия

з Челябинский областной клинический центр онкологии и ядерной медицины, Челябинск, Россия

\begin{abstract}
В России более 10 лет в рамках национальных проектов проводят мероприятия, направленные на преобразование первичной и специализированной, в том числе высокотехнологичной медицинской помощи, усиление роли первичного медико-санитарного звена в целях раннего выявления злокачественных новообразований (ЗНО). Целью исследования было оценить результативность работы женских смотровых кабинетов (СК) в улучшении показателей кумулятивной скорректированной выживаемости (СкВ) больных с ЗНО женских половых органов, выявленных активно с 2005 по 2015 гг. на территории Челябинской области (ЧО). На основе базы данных Популяционного ракового регистра (ПРР) ЧО впервые осуществлены расчеты показателей кумулятивной СкВ больных с ЗНО шейки матки (389), тела матки (373) и яичников (161), выявленных активно в СК ЧО (основная группа), и случаи ЗНО шейки матки (395), тела матки (380) и яичников (163), выявленных в ходе самообращений пациенток (группа контроля). Стадийную структуру активно выявленных случаев ЗНО шейки матки в СК за период 2010-2017 гг. характеризует большой удельный вес І-ІІ стадий по сравнению с остальной популяцией заболевших в 40 - в среднем 50,8 и 46,1\% соответственно, в отношении 3НО тела матки - 85,5 и 82,0\% соответственно, для овариальных раков - в среднем 42,0 и 37,4\% соответственно. Анализ базы данных ПРР 40 за 10-летний период позволил установить увеличение 5-летней кумулятивной СкВ для больных с диагнозом ЗНО шейки матки, выявленных активно в СК по сравнению с контрольной группой - 62,3 и 55,8\% соответственно $(p=0,11)$. В отношении ЗНО тела матки - 82,0\% в основной группе и 70,4\% в группе контроля ( $p=0,001)$. У больных с ЗНО яичников достоверных различий в показателях 5-летней кумулятивной СкВ не отмечено - 47,5\% в основной и 43,2\% в группе контроля ( $p=0,49)$. Таким образом, СК - одна из эффективных организационных моделей вторичной профилактики рака на территории 40.
\end{abstract}

Ключевые слова: рак женских половых органов, раннее выявление, смотровые кабинеты, вторичная профилактика рака

Вклад авторов: А. Г. Бочкова - планирование исследования, подготовка рукописи, сбор данных, анализ и интерпретация данных, анализ литературы; А. С. Доможирова - планирование исследования, анализ и интерпретация данных, редактирование рукописи; И. А. Аксенова - сбор данных, подготовка рукописи.

Соблюдение этических стандартов: исследование одобрено этическим комитетом ЮУгМУ Минздрава России (протокол № 9 от 8 ноября 2018 г.).

$\triangle$ Для корреспонденции: Анна Геннадьевна Бочкова

ул. Доватора, д. 23, г. Челябинск, 454091; annabochkova7496@mail.ru

Статья получена: 23.12.2019 Статья принята к печати: 08.01.2020 Опубликована онлайн: 22.01.2020

DOI: $10.24075 /$ vrgmu.2020.003

\section{EFFICIENCY OF THE GYNECOLOGIC MALIGNANCIES IDENTIFICATION MEASURES AT THE LEVEL OF PRIMARY HEALTH CARE}

Bochkova $A G^{1} \otimes$, Domozhirova $A S^{2}$, Aksenova $I A^{1,3}$

${ }^{1}$ South Ural State Medical University, Chelyabinsk, Russia

${ }^{2}$ Russian Scientific Center of Roentgenoradiology, Moscow, Russia

${ }^{3}$ Clinical Center of Oncology and Nuclear Medicine of Chelyabinsk Region, Chelyabinsk, Russia

In Russia, for more than 10 years, within the framework of national projects, the activities have been carried out aimed at restructuring of the primary and specialized (including high-tech) medical care, as well as strengthening of primary health care directed at early detection of malignant neoplasms. The study was aimed to evaluate the effectiveness of the patient examination rooms for women in improvement of the adjusted cumulative survival of patients with gynecologic malignancies detected actively in 2005-2015 in the Chelyabinsk Region. Using the Population Based Cancer Registry (PBCR) of the Chelyabinsk Region database, the adjusted cumulative survival calculation for patients with cervical cancer (389), uterine corpus cancer (373) and ovarian cancer (161) detected actively in the patient examination rooms of the Chelyabinsk Region (treatment group), as well as for cervical cancer (395), uterine corpus cancer (380) and ovarian cancer (163) detected in patients who sought medical assistance (control group), was performed for the first time. The staging of cervical cancer detected in the examination rooms in 2010-2017 was characterized by the high proportion of I/II stage tumors compared with the rest of patients' population of the Chelyabinsk Region (an average of 50.8 and $46.1 \%$ respectively). For uterine corpus cancer the average proportion was 85.5 and $82.0 \%$ respectively, and for ovarian cancers it was 42.0 and $37.4 \%$ respectively. Analysis of the PBCR of the Chelyabinsk Region for a 10-year period revealed the 5-year adjusted cumulative survival improvement in the actively detected cervical cancer patients compared to the control group (62.3 and 55.8\%) respectively $(p=0.11)$. In patients with uterine corpus cancer, it was $82.0 \%$ for the main group and $70.4 \%$ for the control group $(p=0.001)$. In ovarian cancer patients, no significant differences in the 5 -year adjusted cumulative survival were observed ( $47.5 \%$ in the main group, $43.2 \%$ in the control group) $(p=0.49)$. Thus, the patient examination rooms are the effective model of the cancer secondary prevention available in the Chelyabinsk Region.

Keywords: gynecologic malignancies, early detection, patient examination rooms, secondary prevention of cancer

Author contribution: Bochkova AG — research planning, manuscript writing, data acquisition, data analysis and interpretation, literature analysis; Domozhirova AS — research planning, data analysis and interpretation, manuscript editing; Aksenova IA — data acquisition, manuscript writing.

Compliance with ethical standards: the study was approved by the Ethics Committee of South Ural State Medical University (protocol № 9 dated November 8 , 2018).

$\triangle$ Correspondence should be addressed: Anna G. Bochkova

Dovatora, 23, Chelyabinsk, 454091; annabochkova7496@mail.ru

Received: 23.12.2019 Accepted: 08.01.2020 Published online: 22.01.2020

DOI: 10.24075/brsmu.2020.003 
Злокачественные новообразования $(3 \mathrm{HO})$ - одна из основных проблем, стоящих на повестке дня современной медицины, и основная причина смертности населения развитых и развивающихся стран $[1,2]$. Экономические потери от онкозаболеваний обусловлены значительными затратами на социальное обеспечение и страхование в связи с высокой стоимостью лечения, профилактических и реабилитационных мероприятий, длительной, часто стойкой, утратой трудоспособности, что по праву относит ЗНО к числу значимых проблем современного общества.

Наибольший удельный вес в структуре онкологической заболеваемости у женщин в РФ занимают ЗНО органов репродуктивной системы (38,9\%), из них половину случаев составляют опухоли половых органов.

В 2017 г. в России было зарегистрировано более 26 тыс. новых случаев ЗНО тела матки (3-е место, 7,8\%), более 17,5 тыс. случаев опухолей шейки матки (5-е место, 5,3\%) и более 14,5 тыс. случаев злокачественных опухолей яичников (9-е место, 4,3\%) [3].

Демографический сдвиг, эколого-репродуктивный диссонанс, общее ухудшение состояния здоровья женщин наряду с ростом частоты онкозаболеваний органов репродуктивной системы требуют целенаправленного и безотлагательного принятия решений, касающихся вопросов профилактики, скрининга и ранней диагностики злокачественных новообразований за счет междисциплинарного взаимодействия специалистов из разных областей.

В РФ внедрена в практику трехуровневая система оказания медицинской помощи населению по профилю «Онкология», и первичная медико-санитарная помощь в ней является базовой платформой, на которой реализуется профилактическая направленность системы здравоохранения, формируются основы для выработки у населения мотивации к сохранению и поддержанию высокого уровня здоровья с целью снижения смертности и инвалидизации вследствие заболеваний [4].

Снижение уровня смертности От ЗНО визуальных локализаций женских половых органов всецело зависит от качества и своевременности первичной диагностики [5].

Созданная в начале 1960-х гг. мощная сеть смотровых кабинетов (СK) не раз доказывала свою высокую результативность в активном выявлении ЗНО. Так, удельный вес активно выявленных случаев ЗНО шейки матки среди всех зарегистрированных больных в 1960-70-е гг. достигал 40\% [6].

Рентабельность просилактических осмотров неоднократно подвергали анализу, который показал, что профилактические мероприятия, выявление и лечение больных с предопухолевыми заболеваниями и начальными формами рака требуют значительно меньше государственных затрат, чем лечение больных с клинически выраженными формами [7].

Основная задача женского СК - проведение профилактического осмотра всех женщин (старше 18 лет), обратившихся впервые в течение года в медицинские организации в целях раннего выявления хронических, предопухолевых и опухолевых заболеваний визуальных локализаций. Опухоли наружных локализаций, которые можно обнаружить в ходе профилактических осмотров, составляют 30\% среди всех злокачественных опухолей у лиц обоего пола и почти 40\% - у женщин [8].

Очень важно, чтобы профилактическое обследование в СК носило массовый поточный характер. Так, в популяциях, где качество скрининга и охват населения были высоки, отмечено значительное снижение случаев инвазивного рака шейки матки $[9,10]$.

Кроме того, необходимо понимать, что ни современное оснащение СК, ни высокая квалификация и опыт акушерки, проводящей осмотр, не смогут обеспечить высоких конечных результатов работы, если не определен контингент женщин, подлежащих обследованию, не установлена периодичность осмотров, не отрегулирован поток посещений, не отработана система дообследования и маршрутизации выявленных больных. В этом случае становится актуальной активная позиция руководителей медицинских организаций первичного звена здравоохранения, которые осознают важность профилактического обследования в CK и проводят целенаправленную политику по развитию данного направления.

Цель исследования - оценить увеличение выживаемости больных с ЗНО женских половых органов в Челябинской области (ЧО) за счет использования эффективного пути активного выявления онкологических заболеваний в СК.

\section{ПАЦИЕНТЫ И МЕТОДЫ}

В работе использовали материалы компьютерной базы данных Популяционного ракового регистра (ПРР) 40 о впервые выявленных больных с ЗНО визуальных локализаций женских половых органов, сформированные за период 2005-2017 гг. включительно, а также отчеты о работе СК учреждений здравоохранения муниципальных образований $Ч О$ за указанный период времени.

В основную группу вошли 923 пациентки в возрасте от 20 лет и старше, диагноз ЗНО у которых был выявлен активно в СК $4 О$ в период с 2005 по 2015 г. включительно. При этом распределение по основным локализациям опухолевого процесса выглядело следующим образом: в 389 (42,1\%) наблюдениях были диагностированы ЗНО шейки матки, в 373 (40,4\%) случаях - ЗНО тела матки и 161 (17,4\%) случае - ЗНО яичников соответственно.

Контрольная группа включала 938 больных в возрасте 22 лет и старше, диагноз ЗНО которых установлен в ходе самообращений пациенток за аналогичный период, а именно: в 395 (42,1\%) наблюдениях - рак шейки матки, в $380(40,5 \%)$ и $163(17,4 \%)$ случаях - рак тела матки и яичников соответственно.

Рандомизацию исследуемых групп осуществляли методом стратометрического отбора по совокупности основных прогностических признаков (по возрасту на момент установления диагноза, стадии заболевания, вариантам морфологического строения опухоли) в соответствии с критериями включения и исключения, приведенными ниже.

Критерии включения в группы: 100\%-но морфологически верифицированные случаи 3 НО шейки матки, тела матки и яичников, выявленные активно в СК 4 (для основной группы) либо при самообращении пациенток (для контрольной группы).

Критерии исключения из групп: первично-множественные случаи ЗНО, за исключением случаев сочетания с базально-клеточным раком кожи; отсутствие четких сведений об обстоятельствах выявления онкопатологии; отсутствие сведений о состоянии пациента на конец года; статус «выехавших» пациентов в другие регионы страны по данным ПРР 40.

В основной группе с верифицированным диагнозом ЗНО шейки матки превалировали больные с локализованным опухолевым процессом (общее число больных с I-II стадией 
заболевания составило 199 (51,2\%) человек, в группе контроля - 199 (50,4\%)) (р > 0,05) (табл. 1).

Стадийное распределение впервые выявленных случаев ЗНО тела матки в обеих исследуемых группах демонстрирует высокий удельный вес выявленных I-II стадий заболеваний: 303 (81,2\%) в основной группе и 303 $(79,7 \%)$ в группе контроля ( $p>0,05)$, что можно объяснить особенностями клинической картины заболевания, заставляющей больных рано обращаться к врачам, особенно в пре-и менопаузальном возрасте (табл. 2).

В отношении ЗНО яичников в обеих исследуемых группах доминировало наибольшее процентное соотношение больных с III-IV стадиями заболевания: 96 (59,6\%) в основной и 96 (58,9\%) в контрольной группах; различия были статистически недостоверными ( $>$ > 0,05) (табл. 3).

Возраст больных ЗНО шейки матки в основной группе варьировал от 23 до 92 лет, средний возраст составил $51,6 \pm 14,2$ лет, и в группе контроля от 20 до 88 лет, средний возраст $-51,4 \pm 14,0$ лет ( $p=0,81)$. Необходимо отметить, что именно женщины возрастной категории 40-59 лет составляют основной контингент обследуемых в СК, тогда как лица 39 лет и младше составляют только одну четвертую часть от общего числа осмотренных. Такой неудовлетворительный охват молодых пациенток профилактическими осмотрами в итоге отражается в увеличении в 1,5 раза выявления инвазивных форм рака шейки матки на территории 40 в данной возрастной группе за последние 10 лет.

Возраст больных с диагнозом ЗНО тела матки в основной группе находился в диапазоне 31-86 лет, средний возраст составил 62,2 \pm 9,7 лет и в диапазоне 35-85 лет в контрольной группе, средний возраст $61,9 \pm$ 9,7 лет; различия по возрастам были статистически недостоверными ( $p=0,72)$. Наибольший удельный вес заболеваемости раком эндометрия пришелся на возрастную когорту женщин 60-69 лет - 130 (34,9\%) человек в основной группе и 136 (35,8\%) в группе контроля.

Возрастной интервал больных с ЗНО яичников в основной группе составил 20-80 лет, средний возраст 57,3 \pm 13,5 лет, в группе сравнения - 20-83 лет, средний возраст $57,1 \pm 13,0$ лет $(p=0,90)$.

Прогностически важным фактором в оценке выживаемости онкологических больных является морфологический тип первичной опухоли, который был верифицирован у всех больных сравниваемых групп. При статистическом анализе в обеих группах с диагнозом ЗНО шейки матки, ЗНО тела матки и яичников по основным гистологическим типам опухолей достоверных различий выявлено не было ( $p>0,05)$.

На основе базы данных ПРР 40 впервые осуществлены расчеты показателей СкВ больных с ЗНО шейки матки, тела матки и яичников с учетом обстоятельств их выявления. Расчеты показателей выживаемости проведены по классическим методам анализа данных на популяционном уровне. Для расчета выживаемости на популяционном уровне использовали актуариальный (динамический) метод с учетом вероятной продолжительности жизни и использованием повозрастных таблиц смертности населения на каждый период расчета показателя. За начало отсчета взята дата начала заболевания (дата установления диагноза), рекомендуемая для оценки выживаемости на популяционном уровне. Если показатели наблюдаемой выживаемости рассчитывают с учетом всех причин смерти онкологического пациента, по причине чего они являются ориентировочными, то показатели скорректированной выживаемости учитывают случаи смерти онкологических больных только по основному заболеванию, при этом умершие от интеркуррентных

Таблица 1. Распределение по стадиям заболевания в основной группе (СК) и группе контроля (КГ) случаев ЗНО шейки матки (С53) в соответствии с классификацией FIGO (2009 г.)

\begin{tabular}{|c|c|c|c|c|c|c|c|c|c|c|c|c|c|}
\hline \multirow{2}{*}{\multicolumn{2}{|c|}{ Группы сравнения }} & \multicolumn{12}{|c|}{ Стадия заболевания } \\
\hline & & 1 & IA & IB & II & IIA & IIB & III & IIIA & IIIB & IV & IVA & IV B \\
\hline CK & Абс. & 28 & 22 & 33 & 27 & 8 & 81 & 25 & 2 & 141 & 14 & 4 & 4 \\
\hline$(n-389)$ & $\%$ & 7,2 & 5,7 & 8,5 & 6,9 & 2,1 & 20,8 & 6,4 & 0,5 & 36,2 & 3,6 & 1 & 1 \\
\hline $\mathrm{K} \Gamma$ & Абс. & 30 & 23 & 28 & 33 & 4 & 81 & 32 & 4 & 136 & 16 & 4 & 4 \\
\hline$(n-395)$ & $\%$ & 7,6 & 5,8 & 7,1 & 8,4 & 1 & 20,5 & 8,1 & 1 & 34,4 & 4,1 & 1 & 1 \\
\hline
\end{tabular}

Таблица 2. Распределение по стадиям заболевания в основной группе (СК) и группе контроля (КГ) случаев ЗНО тела матки (С54) в соответствии с классификацией FIGO (2009 г.)

\begin{tabular}{|c|c|c|c|c|c|c|c|c|c|c|c|c|c|c|}
\hline \multirow{2}{*}{\multicolumn{2}{|c|}{ Группы сравнения }} & \multicolumn{13}{|c|}{ Стадия заболевания } \\
\hline & & \multirow{2}{*}{$\begin{array}{c}1 \\
99\end{array}$} & \multirow{2}{*}{$\begin{array}{l}\text { IA } \\
52\end{array}$} & \multirow{2}{*}{$\begin{array}{l}\text { I B } \\
79\end{array}$} & \multirow{2}{*}{$\begin{array}{l}\text { II } \\
14\end{array}$} & \multirow{2}{*}{$\begin{array}{l}\text { IIA } \\
45\end{array}$} & \multirow{2}{*}{$\begin{array}{l}\text { IIB } \\
14 \\
\end{array}$} & \multirow{2}{*}{$\begin{array}{l}\text { III } \\
24\end{array}$} & \multirow{2}{*}{$\begin{array}{l}I I A \\
12\end{array}$} & \multirow{2}{*}{$\begin{array}{c}\text { IIIB } \\
13 \\
\end{array}$} & \multirow{2}{*}{$\begin{array}{c}\text { IIIC } \\
4\end{array}$} & \multirow{2}{*}{$\frac{\text { IV }}{2}$} & \multirow{2}{*}{$\begin{array}{l}\text { IVA } \\
12 \\
\end{array}$} & \multirow{2}{*}{$\begin{array}{c}\text { IV B } \\
3 \\
\end{array}$} \\
\hline CK & Абс. & & & & & & & & & & & & & \\
\hline$(n-373)$ & $\%$ & 26,5 & 13,9 & 21,2 & 3,8 & 12,1 & 3,8 & 6,4 & 3,2 & 3,5 & 1,1 & 0,5 & 3,2 & 0,8 \\
\hline $\mathrm{K} \Gamma$ & Абс. & 95 & 60 & 78 & 12 & 47 & 11 & 26 & 18 & 14 & 1 & 2 & 14 & 2 \\
\hline$(n-380)$ & $\%$ & 25 & 15,8 & 20,5 & 3,2 & 12,4 & 2,9 & 6,8 & 4,7 & 3,7 & 0,3 & 0,5 & 3,7 & 0,5 \\
\hline
\end{tabular}

Таблица 3. Распределение по стадиям заболевания в основной группе (СК) и группе контроля (КГ) случаев ЗНО яичников (С56) в соответствии с классификацией FIGO (2009 г.)

\begin{tabular}{|c|c|c|c|c|c|c|c|c|c|c|c|c|c|c|}
\hline \multirow{2}{*}{\multicolumn{2}{|c|}{ Группы сравнения }} & \multicolumn{13}{|c|}{ Стадия заболевания } \\
\hline & & I & $\mathrm{IA}$ & $\mathrm{IB}$ & IC & II & $\| A$ & IIB & IIC & III & IIIA & IIIB & $I I I C$ & IV \\
\hline CK & Абс. & 21 & 14 & 1 & 17 & 3 & 2 & 5 & 2 & 26 & 4 & 3 & 31 & 32 \\
\hline$(n-161)$ & $\%$ & 13 & 8,7 & 0,6 & 10,6 & 1,9 & 1,2 & 3,1 & 1,2 & 16,1 & 2,5 & 1,9 & 19,3 & 19,9 \\
\hline $\mathrm{K} \Gamma$ & Абс. & 21 & 16 & 1 & 16 & 4 & 2 & 6 & 1 & 25 & 3 & 3 & 34 & 31 \\
\hline$(n-163)$ & $\%$ & 12,9 & 9,8 & 0,6 & 9,8 & 2,5 & 1,2 & 3,7 & 0,6 & 15,3 & 1,8 & 1,8 & 20,9 & 19 \\
\hline
\end{tabular}


заболеваний приравниваются к выбывшим из-под наблюдения; все показатели выражают в \% [11, 12]. Исчисление показателей выживаемости и их средних ошибок осуществляли с помощью математической части программного обеспечения ПРР чО. Разницу считали значимой при $p<0,05$.

\section{РЕЗУЛЬТАТЫ ИССЛЕДОВАНИЯ}

В период с 2005 по 2017 г. в 27 муниципальных районах и 16 городских округах 40 активно функционировало 167 CK из 176 развернутых. Общее число женских СК составило 104. Кроме того, в муниципальных районах функция СК возложена и на фельдшерско-акушерские пункты, которые совмещают в себе работу женского и мужского СК.

Работу в женских СК осуществляет средний медицинский персонал (фельдшер, акушерка), прошедший специальную подготовку по онкологии и имеющий соответствующий сертификат, или врач акушер-гинеколог или дерматовенеролог, прошедший повышение квалификации по онкологии в объеме не менее 72 учебных часов. Согласно требованиям, обследование в СК должно нацеливать медицинских работников на выявление $3 \mathrm{HO}$ визуальных локализаций как у мужчин, так и у женщин, и не «носить» выборочного характера обследования по отдельным органам и системам.

Востребованность и эффективность работы этого вида технологий вторичной профилактики ЗНО обусловлень следующими составляющими:

- доступность оказываемой медицинской помощи: работа в две смены, осмотр в первый день посещения лечебно-профилактического учреждения, отсутствие очередности и надобности заблаговременного бронирования талонов для осмотра;

- четкое соблюдение принципов маршрутизации пациентов с выявленной патологией и в случае выявления ЗНО, передача пациента «из рук в руки» в день обращения к врачу соответствующей специальности или онкологу с приоритетом первоочередности обследования;

- обязательный отбор у женщин мазков с цервикального канала и шейки матки и последующее направление в цитологическую лабораторию, их учет и регистрация, а также мониторирование числа неинформативных мазков.

С 2007 по 2017 г. женские СК 40 посетило более 5 млн. женщин (5 136 098) в возрасте старше 20 лет с целью осмотра и проведения цитологического скрининга для выявления предопухолевых, хронических заболеваний и онкопатологии, т. е. в среднем 49,5\% от числа женщин старше 20 лет, проживающих на данной территории. Таким образом, создается некий «резерв» в среднем из 50,5\% лиц, которые обращаются за медицинской помощью гораздо реже, чем раз в год, а значит не могут быть в полной мере охвачены профилактическими мероприятиями, в том числе осмотрами в СК.

За время своей работы СК доказали высокую результативность, что находит отражение в активном выявлении не только общего числа ЗНО визуальных локализаций женских половых органов, но и в общем количестве выявленных случаев хронических и предопухолевых заболеваний. Так, в 2017 г. работниками СК диагностировано 97367 всех случаев заболеваний у женщин, что на 53,7\% больше по сравнению с 2007 г. В структуре выявленных заболеваний наибольший удельный вес имеют хронические заболевания: в 2017 г. -
66805 (68,61\%) случаев, в 2007 г. - 40106 (63,3\%); прирост за 10-летний период наблюдения составил 66,6\%. Выявляемость хронических заболеваний (\% от числа осмотренных в СК) в среднем составила $11,2 \%$. Второе место по выявляемости в СК занимают предопухолевые заболевания - 29885 (30,7\%) случаев в 2017 г., 22801 (36,0\%) - в 2007 г.; прирост за указанный период составил $31,1 \%$, выявляемость в среднем по области - 5,9\% от числа осмотренных. Третье место по выявляемости в СК занимают ЗНО: в 2017 г. - 677 случаев ЗНО (12,0 на 10 тыс. осмотренных), что составляет 7,5\% от всех выявленных 3 НО у женщин в 4О, по сравнению с 2007 г. прирост абсолютного числа выявленных случаев ЗНО увеличился в 1,5 раза (на 50,8\%).

В период с 2007 по 2017 гг. специалистами CK было выявлено 548 случаев 3НО тела матки (8,6\% от общего числа эндометриоидного рака в области), 567 случаев ЗНО шейки матки (в среднем 12,9\% от числа выявленных случаев ЗНО шейки матки в области) и 254 случаев $3 \mathrm{HO}$ яичников (6,4\% от общего числа овариальных раков в области). Удельный вес выявленных случаев ЗНО тела матки на I-II стадиях в среднем за период 2010-2017 гг. составил 85,5\%, ЗНО шейки матки - 50,8\%, а $3 \mathrm{HO}$ яичников - 42,0\%, что выше аналогичных показателей по области - 82,0\%, 46,1\% и 37,4\% соответственно (рис. 1-3).

При оценке анализа работы CK большое внимание уделяли показателям мощности работы (загруженности), которые исчисляются, исходя из регламентированной длительности рабочего дня СК - 7 ч (смена) с осмотром не менее четырех пациентов в час 252 рабочих дня в году.

В 2007 г. в период становления работы СК показатель загруженности достигал 51,4\% и, начиная с 2009 г., с открытием нового числа СК, отмечено увеличение мощности работы (загруженности) до 77,9\% в 2017 г. Нами были произведены расчеты относительно возможного количества выявленных случаев ЗНО при идеальных условиях - 100\%-й загрузке CK. Полученные данные позволили сделать заключение о том, что при полной загрузке женских CK в области в период 2007-2017 гг. дополнительно было бы выявлено 3438 случаев ЗНО вместо существующих 6876, т. е. на 50,0\% больше от исходного уровня. Из них удельный вес $3 \mathrm{HO} \mathrm{I-ІІ} \mathrm{стадий} \mathrm{оказался} \mathrm{бы}$ равным 7448 случаям, вместо 4986 имеющихся. Методом наименьших квадратов аппроксимирована имеющаяся прямая зависимость между количеством осмотренных пациенток и количеством выявленных случаев $3 \mathrm{HO}$ в CK 40 за 10-летний период наблюдения, коэффициент детерминации составил 0,591, что демонстрирует высокую корреляцию модели с реальными данными (коэффиициент корреляции $r=0,77 ; p=0,007)$.

С применением цитологического скрининга в СК было обследовано от 79,6\% в 2007 г. до 94,7\% женщин в 2017 г., при этом охват цитологическими обследованиями должен стремиться к показателю, равному 100\%. Общее количество взятых цитологических мазков в период с 2007 по 2017 г. составило более 4,5 млн (в среднем более 410 тыс. мазков в год).

Важнейшим качественным критерием оценки эффективности противораковых мероприятий служит показатель выживаемости онкологических больных, который в том числе отражает адекватность стадирования всех выявленных случаев ЗНО [13].

В ходе исследования удалось показать преимущество в показателях 5-летней кумулятивной СкВ для больных с диагнозом ЗНО шейки матки, выявленных активно в СК по 
сравнению с контрольной группой - 62,3 против 55,8\% $(p=0,11)$ (рис. 4).

В отношении ЗНО тела матки были получены достоверные различия в показателях 5-летней выживаемости: 82,0 \pm 2,6 - в основной группе и 70,4 \pm 2,6 - в группе контроля $(p=0,001)$. На рис. 5 видно, как с течением времени расходятся кривые выживаемости.

У больных с овариальными раками достоверных различий в показателях 5-летней кумулятивной СкВ не отмечено: $47,5 \%$ - в основной и 43,2\% - в группе контроля $(p=0,49)$ (рис. 6). Многие авторы сходятся во мнении, что несмотря на высокую чувствительность большинства современных методов диагностики их специфичность недостаточна для дифференцировки доброкачественных и злокачественных процессов в яичниках, а проведение диагностических мероприятий всем без исключения женщинам (в том числе не входящим в группу высокого риска) в итоге оказывает минимальное влияние на показатели летальности [14-16].

\section{ОБСУЖДЕНИЕ РЕЗУЛЬТАТОВ}

Совершенствование системы раннего выявления опухолей необходимо начинать с наиболее доступных для этого визуальных локализации рака, диагностика которых не требует специального дорогостоящего диагностического оборудования и углубленных знаний по вопросам онкологии у медицинских работников первичного звена здравоохранения.

Несмотря на то что просрилактические осмотры играют значительную роль в диагностике ЗНО, за последние 10 лет в РФ число СК увеличилось на 48\% и в 2017 г. составило 4989, т. е. в среднем 58 СК на каждый из 85 существующих субъектов РФ (для сравнения в одной $4 О$ в 2017 г. число

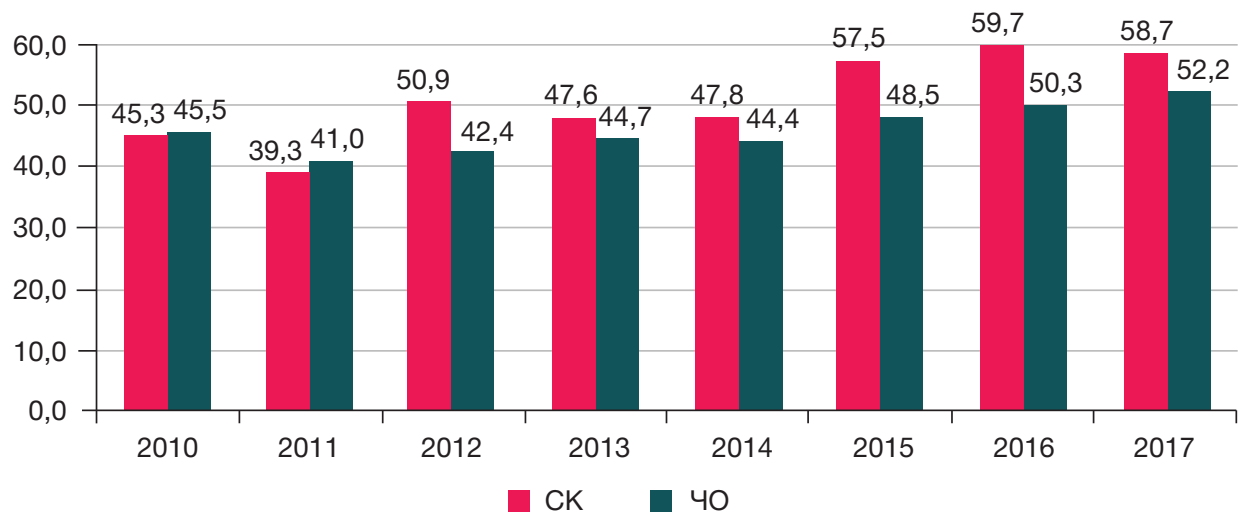

Рис. 1. Динамика удельного веса І-ІІ стадий ЗНО шейки матки (С53), выявленных активно в смотровых кабинетах в сравнении со среднеобластным показателем по 40 в 2010-2017 гг., в \%

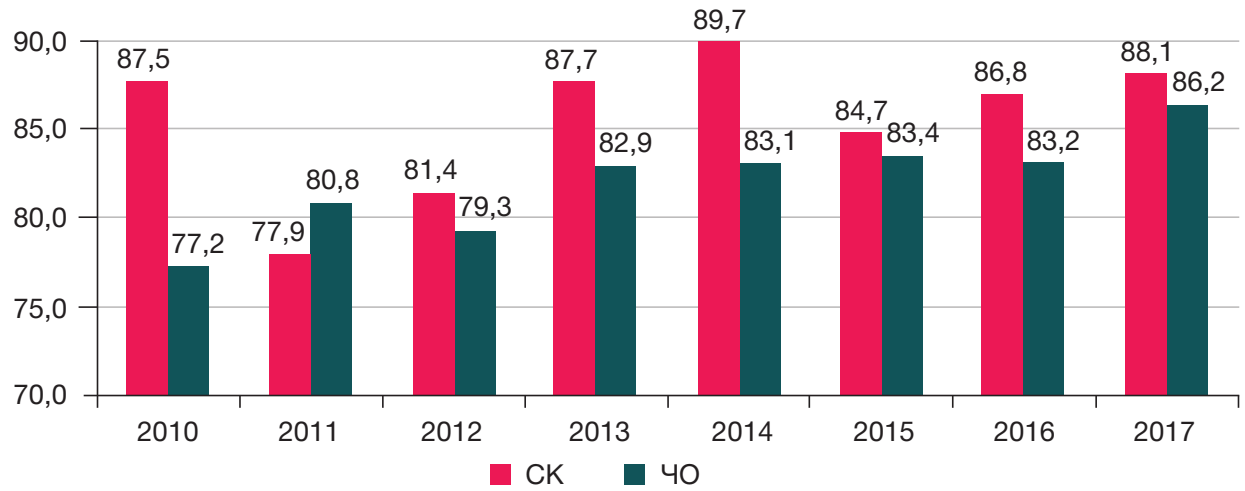

Рис. 2. Динамика удельного веса I-ІІ стадий ЗНО тела матки (C54), выявленных активно в смотровых кабинетах в сравнении со среднеобластным показателем по 40 в 2010-2017 гг., в \%

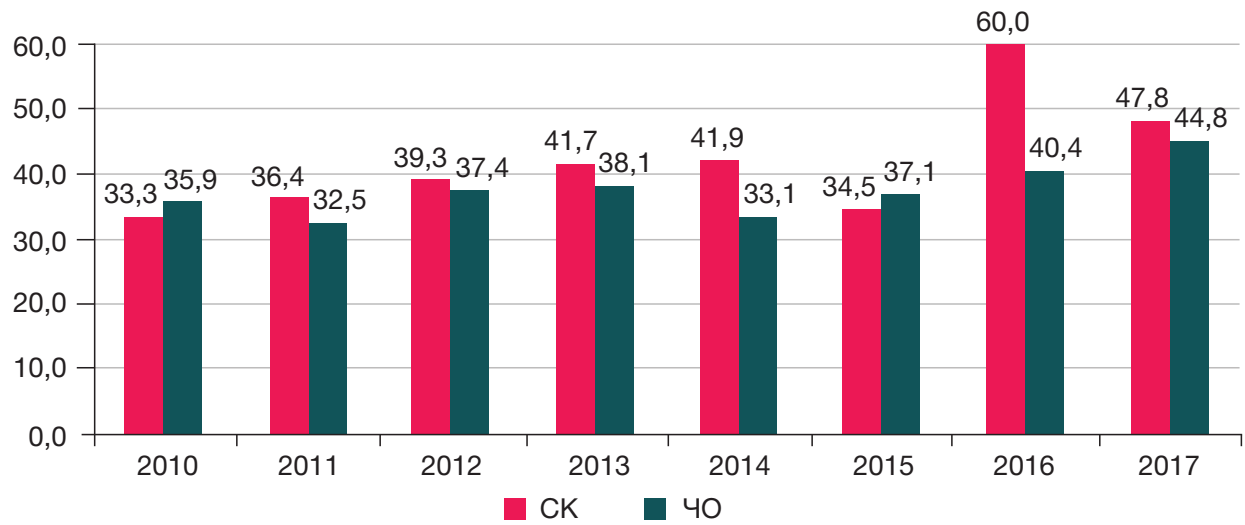

Рис. 3. Динамика удельного веса I-ІІ стадий ЗНО яичников (С56), выявленных активно в смотровых кабинетах в сравнении со среднеобластным показателем по ЧО в 2010-2017 гг., в \% 
СК достигло 176), при этом количество работающих в две смены оказалось равным только 20\%. Недостаточный охват населения просилактическими осмотрами в итоге отражается на низком удельном весе активно выявленных случаев ЗНО в целом - 25,8\% в 2017 г. в РФ. Удельный вес ЗНО шейки матки, выявленных активно в РФ, несмотря на визуально доступную локализацию в 2017 г. был равен 42,2\%; значительно ниже доля больных на профилактических осмотрах при раке тела матки (29,1\% в 2017 г.) и яичников (18,4\% в 2017 г.). Как следствие, каждый второй и третий случаи рака яичников и шейки матки в РФ диагностируют на III-IV стадиях процесса - 58,5 и 32,4\% соответственно в 2017 г., что приводит к низким показателям выживаемости в этих группах, несмотря на арсенал задействованных комплексных методов лечения [17]. Именно поэтому в настоящее время назрела настоятельная потребность реализации профилактических программ национального масштаба, направленных на раннее выявление и адекватное лечение предотвратимых форм $3 \mathrm{HO}$.

Анализ базы данных ПРР 40 за 10-летний период наблюдения позволил установить, что показатели 5-летней кумулятивной СкВ с ЗНО шейки матки, выявленными активно в СК, выглядят более выигрышными по сравнению с остальной популяцией заболевших - 62,3 и 55,8\% соответственно ( $p=0,11)$. Подобная тенденция прослеживается и в отношении опухолей невизуальных локализаций, таких как ЗНО тела матки и яичников 82,0 против $70,4 \%(p=0,001)$ и 47,5 против 43,2\% в соответственно $(p=0,49)$.

Такая гетерогенная группа опухолей, как овариальный рак, уже на протяжении более 30 лет ассоциируется с незначительными успехами по отдаленным результатам лечения с показателями 5-летней выживаемости, не превышающими 50\%-й порог (48,4\%) [18]. Во многом это связано с отсутствием на сегодняшний день надежных скрининговых программ, позволяющих решить вопросы ранней диагностики рака яичника.

В целом, на высокие показатели работы СК оказывает влияние массовый поточный характер проводимых профилактических обследований в них с осмотром не менее четырех пациентов в час на протяжении полного рабочего дня поликлиники, в две смены. Именно такой объем нагрузки позволяет отсортировать из общей массы обследуемых когорту лиц с клинически неманифестными формами патологии для последующего

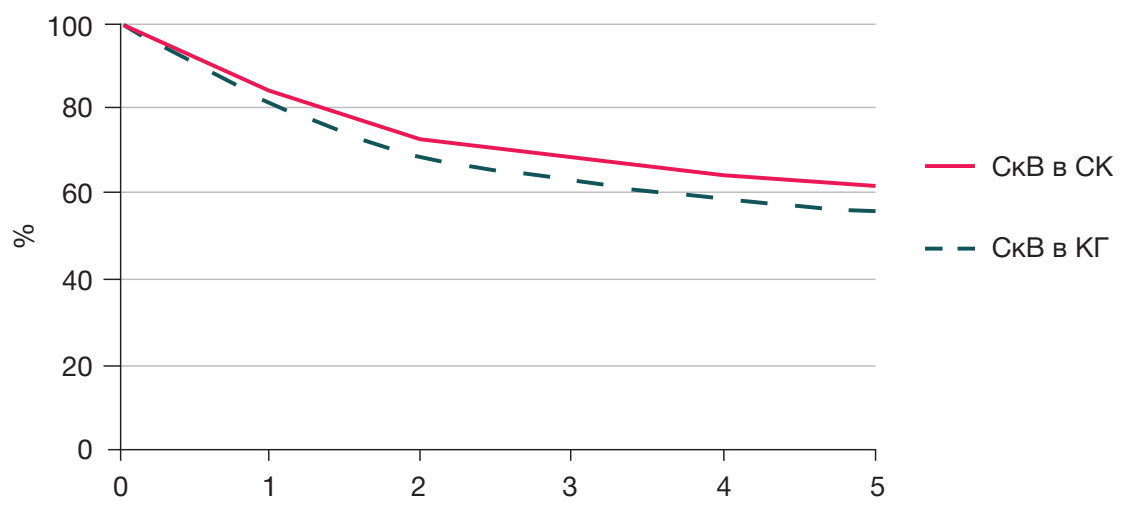

\begin{tabular}{|c|c|c|c|c|c|c|}
\hline Выживаемость & Группы & 1-й год & 2-й год & 3-й год & 4-й год & 5 лет \\
\hline \multirow{2}{*}{ Скорректированная } & СК & 84,1 & 72,9 & 68,6 & 64,4 & 62,3 \\
\cline { 2 - 8 } & КГ & 81,3 & 68,5 & 63,2 & 59,0 & 55,8 \\
\hline
\end{tabular}

Рис. 4. Кумулятивная скорректированная выживаемость больных с ЗНО шейки матки (С53) в основной и контрольной группах (БД пРР ЧО, 2005-2015 гт.), в \%

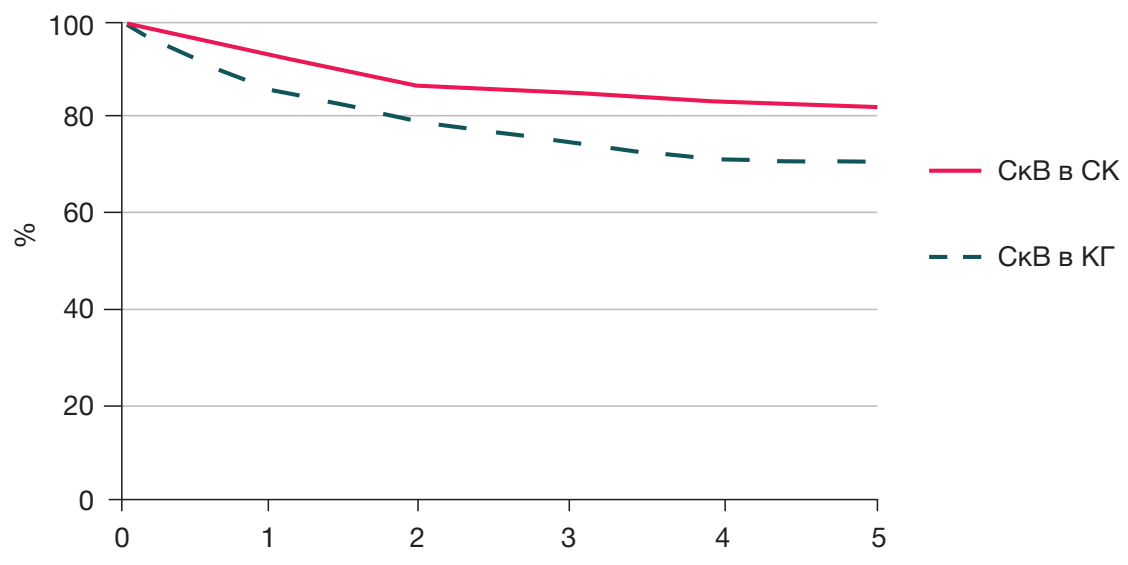

\begin{tabular}{|c|c|c|c|c|c|c|}
\hline Выживаемость & Группы & 1-й год & 2-й год & 3-й год & 4-й год \\
\hline \multirow{2}{*}{ Скорректированная } & СК & 92,4 & 86,8 & 84,9 & 83,0 \\
\cline { 2 - 7 } & КГ & 85,8 & 79,8 & 75,1 & 71,2 \\
\hline
\end{tabular}

Рис. 5. Кумулятивная скорректированная выживаемость больных с ЗНО тела матки (С54) в основной и контрольной группах (БД ПРР чО, 2005-2015 гт.), в \% 


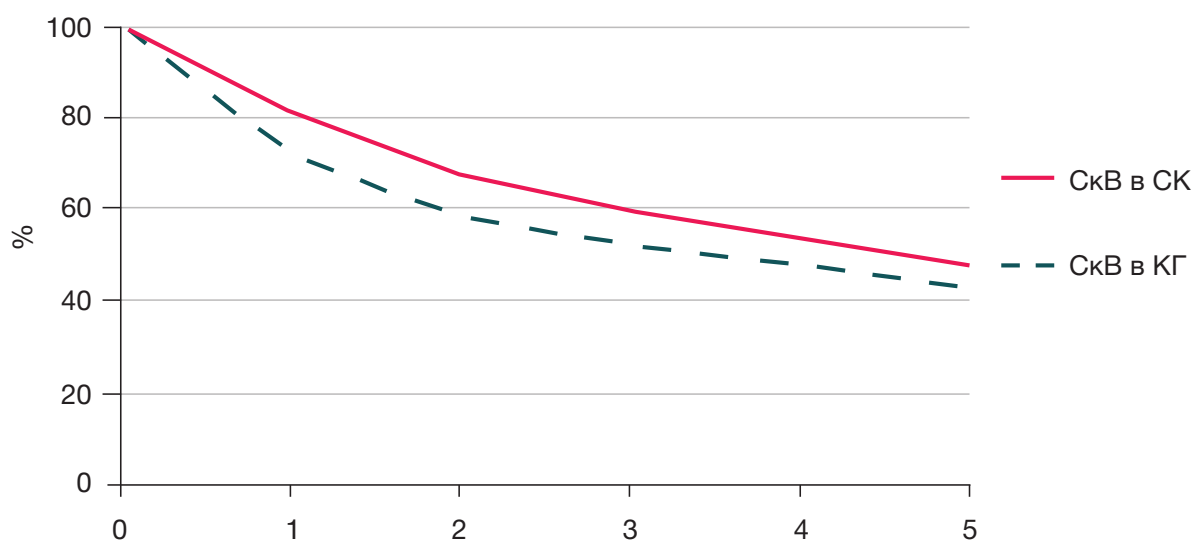

\begin{tabular}{|c|c|c|c|c|c|c|}
\hline Выживаемость & Группы & 1-й год & 2-й год & 3-й год & 4-й год & 5 лет \\
\hline \multirow{2}{*}{ Скорректированная } & СК & 80,6 & 67,2 & 60,1 & 54,1 & 47,5 \\
\cline { 2 - 8 } & КГ & 72,2 & 58,5 & 52,0 & 48,5 & 43,2 \\
\hline
\end{tabular}

Рис. 6. Кумулятивная скорректированная выживаемость больных с ЗНО яичников (С56) в основной и контрольной группах (БД ПРР ЧО, $2005-2015$ гг.), в \%

углубленного обследования. Подобная задача становится невыполнимой для узкого специалиста по причине того, что вследствие особенностей менталитета наши пациенты планируют визит к профильному специалисту лишь с перечнем определенных, присущих квалификации конкретного специалиста вопросов. Усложняет работу и система заблаговременного бронирования талонов к врачам, что заставляет пациентов подстраиваться и выбирать свободное время для этих визитов. В то время как в СК предлагают пройти полноценный осмотр в первый день посещения медицинского учреждения.

По результатам такого осмотра, пациенты с подозрением на онкопатологию с первых шагов попадают в поле пристального внимания специалистов медицинской организации с правом первоочередности углубленного обследования, при этом сроки предполагаемого углубленного обследования должны укладываться в строгие временные рамки, а именно 10 дней с момента выставления подозрения. Такие пациенты, в отличие от прочих, без задержек попадают в онкологические учреждения в случае диагностирования ЗНО, а значит своевременно получают специализированную онкологическую помощь в полном объеме.

\section{ВЫВОДЫ}

Дальнейшая проработка программ по улучшению посещаемости СК области, а также внедрение предложенного тактического алгоритма в работу первичного медико-санитарного звена акушерско-гинекологической службы по раннему выявлению фоновых, предраковых и злокачественных новообразований в будущем позволят повысить показатели выявления ЗНО на более ранних стадиях, а значит минимизировать затраты на дальнейшее лечение и реабилитацию таких пациенток, и самое главное улучшить показатели выживаемости для этой группы больных.

\section{Литература}

1. Сушинская Т. В., Жорданиа К. И., Паяниди Ю.Г. Аналитические аспекты онкологических заболеваний женского населения России. Онкогинекология. 2015; (3): 40-3.

2. Global Cancer Facts \& Figures. 3rd edition. Atlanta, Georgia: American Cancer Society. 2015; 61 p.

3. Каприн А. Д., Старинский В. В., Петрова Г. В., редакторы. Злокачественные новообразования в России в 2017 году (заболеваемость и смертность). М.: МНИОИ им. П. А. Герцена филиал ФГБУ «НМИЦрадиологии» Минздрава России, 2018; $250 \mathrm{c}$.

4. Александрова Л. М., Старинский В. В., Каприн А. Д., Самсонов Ю. В. Профилактика онкологических заболеваний как основа взаимодействия онкологической службы с первичным звеном здравоохранения. Исследования и практика в медицине. 2017; (4): 74-80.

5. Мошуров И. П., Кравец Б. Б., Коротких Н. В. Организационные резервы снижения смертности от рака шейки матки. Онкогинекология. 2014; (4): 28-33.

6. Кривонос О. В., Чиссов В. И., Старинский В. В., Александрова Л. М. Роль и задачи смотрового кабинета поликлиники как этапа в организации профилактических мероприятий, направленных на совершенствование онкологической помощи населению: методические рекомендации. М., 2010. Доступно по ссылке:

http://www.oncology.ru/service/organization/exam_room.pdf (дата обращения: 10.11.2019).

7. Доможирова А. С., Важенин А. В. Вторичная профилактика рака в системе регионального здравоохранения. М.: Изд-во PAMH, 2012; 192 C.

8. Шубин Б. М., Винокур М. Л., Попова А. А. Выявление опухолевых заболеваний в смотровом кабинете. Ленинград: Медицина, 1980; 208 с.

9. ACOG Practice Bulletin Number 131: Screening for cervical cancer. Obstet Gynecol. 2012; 120 (5): 1222-38.

10. Ronco G, Anttila A. Cervical cancer screening in Europe changes over the last 9 years. Eur J Cancer. 2009; 45 (15): 2629-31. DOI: 10.1016/j.ejca.2009.07.021.

11. Петрова Г. В., Грецова О. П., Каприн А. Д., Старинский В. В. Характеристика и методы расчета медико-статистических показателей, применяемых в онкологии. М.: ФГБУ МНИОИ им. П. А. Герцена Минздрава РФ, 2014; 40 c.

12. Мерабишвили В. М. Онкологическая статистика (традиционные методы, новые информационные технологии): Руководство для врачей. Изд. 2-е, доп. СПб., 2015; 248 с.

13. Мерабишвили В. М. Выживаемость онкологических больных. СПб., 2011; 329 c.

14. Урманчеева А. Ф., Кутушева Г. Ф., Ульрих Е. А. Опухоли яичника 
(клиника, диагностика и лечение). СПб.: Изд-во Н-Л, 2012; 68 с.

15. Jacobs IJ, Menon U, Ryan A, Gentry-Maharaj A, Burnell M, Kalsi JK, et al. Ovarian cancer screening and mortality in the UK Collaborative Trial of Ovarian Cancer Screening (UKCTOCS): a randomised controlled trial. Lancet. 2016; 387 (10022): 945-56. PubMed PMID: 26707054.

16. Johnson N. Two large randomised trials show ovarian cancer screening has minimal impact on survival. BJOG. 2016; 125 (5): 524-5.
17. Каприн А. Д., Старинский В. В., Петрова Г. В., редакторы. Состояние онкологической помощи населению России в 2017 году. М.: МНИОИ им. П.А. Герцена - филиал ФГБУ «НМИЦрадиологии» Минздрава России, 2018; 236 с.

18. Howlader N, Noone AM, Krapcho M, Miller D, Brest A, YuM, et al. SEER Cancer Statistics Review, 1975-2016, National Cancer Institute. Bethesda, MD. Available from: https://seer.cancer.gov/ csr/1975_2016/sections.html.

\section{References}

1. Sushinskaya TV, Zhordania KI, Payanidi YuG. Analiticheskie aspekty onkologicheskikh zabolevaniy zhenskogo naseleniya Rossii. Onkoginekologiya. 2015; (3): 40-3. Russian.

2. Global Cancer Facts \& Figures. 3rd edition. Atlanta, Georgia: American Cancer Society. 2015; 61 p.

3. Kaprin AD, Starinskiy W, Petrova GV, redaktory. Zlokachestvennye novoobrazovaniya $\vee$ Rossii $\vee 2017$ godu (zabolevaemost' i smertnost'). M.: MNIOI im. P.A. Gertsena - filial FGBU «NMICradiologii» Minzdrava Rossii, 2018; 250 s. Russian.

4. Aleksandrova LM, Starinskiy VV, Kaprin AD, Samsonov YuV. Profilaktika onkologicheskikh zabolevaniy kak osnova vzaimodeystviya onkologicheskoy sluzhby s pervichnym zvenom zdravookhraneniya. Issledovaniya i praktika v meditsine. 2017; (4): 74-80. Russian.

5. Moshurov IP, Kravets BB, Korotkikh NV. Organizatsionnye rezervy snizheniya smertnosti ot raka sheyki matki. Onkoginekologiya. 2014; (4): 28-33. Russian.

6. Krivonos OV, Chissov VI, Starinskiy WV, Aleksandrova LM. Rol'i zadachi smotrovogo kabineta polikliniki kak etapa $v$ organizatsii profilakticheskikh meropriyatiy, napravlennykh na sovershenstvovanie onkologicheskoy pomoshchi naseleniyu: metodicheskie rekomendatsii. M., 2010. Available from: http:// www.oncology.ru/service/organization/exam room.pdf (data obrascheniya: 10.11.2019). Russian.

7. Domozhirova AS, Vazhenin AV. Vtorichnaya profilaktika raka $\vee$ sisteme regional'nogo zdravookhraneniya. M.: Izd-vo RAMN, 2012; 192 s. Russian.

8. Shubin BM, Vinokur ML, Popova AA. Vyyavlenie opukholevykh zabolevaniy v smotrovom kabinete. Leningrad: Meditsina, 1980; 208 s. Russian.

9. ACOG Practice Bulletin Number 131: Screening for cervical

cancer. Obstet Gynecol. 2012; 120 (5): 1222-38.

10. Ronco G, Anttila A. Cervical cancer screening in Europe changes over the last 9 years. Eur J Cancer. 2009; 45 (15): 2629-31. DOI: 10.1016/i.ejca.2009.07.021.

11. Petrova GV, Grecova OP, Kaprin AD, Starinskij W. Harakteristika i metody rascheta mediko-statisticheskih pokazatelej, primenyaemyh v onkologii. M.: FGBU MNIOI im. P.A. Gercena Minzdrava RF, 2014; 40 s.

12. Merabishvili VM. Onkologicheskaya statistika (tradicionnye metody, novye informacionnye tekhnologii): Rukovodstvo dlya vrachej. Izd. 2-e, dop. SPb., 2015; 248 s.

13. Merabishvili VM. Vyzhivaemost' onkologicheskih bol'nyh. SPb., 2011; $329 \mathrm{~s}$.

14. Urmancheeva AF, Kutusheva GF, Ul'rih EA. Opuholi yaichnika (klinika, diagnostika i lechenie). SPb.: Izd-vo N-L, 2012; 68 s.

15. Jacobs IJ, Menon U, Ryan A, Gentry-Maharaj A, Burnell M, Kalsi JK, et al. Ovarian cancer screening and mortality in the UK Collaborative Trial of Ovarian Cancer Screening (UKCTOCS): a randomised controlled trial. Lancet. 2016; 387 (10022): 945-56. PubMed PMID: 26707054

16. Johnson $\mathrm{N}$. Two large randomised trials show ovarian cancer screening has minimal impact on survival. BJOG. 2016; 125 (5): $524-5$.

17. Kaprin AD, Starinskij W, Petrova GV, redaktory. Sostoyanie onkologicheskoj pomoshchi naseleniyu Rossii v 2017 godu. M.: MNIOI im. P.A. Gercena - filial FGBU «NMICradiologiii Minzdrava Rossii, 2018; 236 s.

18. Howlader N, Noone AM, Krapcho M, Miller D, Brest A, Yu M, et al. SEER Cancer Statistics Review, 1975-2016, National Cancer Institute. Bethesda, MD. Available from: https://seer.cancer.gov/ csr/1975_2016/sections.html. 